\title{
A MAD Q-set
}

by

Arnold W. Miller (Madison, WI)

\begin{abstract}
A MAD (maximal almost disjoint) family is an infinite subset $\mathcal{A}$ of the infinite subsets of $\omega=\{0,1,2, \ldots\}$ such that any two elements of $\mathcal{A}$ intersect in a finite set and every infinite subset of $\omega$ meets some element of $\mathcal{A}$ in an infinite set. A Q-set is an uncountable set of reals such that every subset is a relative $G_{\delta}$-set. It is shown that it is relatively consistent with ZFC that there exists a MAD family which is also a Q-set in the topology it inherits as a subset of $P(\omega)=2^{\omega}$.
\end{abstract}

In this paper we answer a question of Hrušák by showing that it is consistent that there exists a maximal almost disjoint family $\mathcal{A} \subseteq[\omega]^{\omega}$ which is also a Q-set. The reference Hrušák [6] contains some related problems. A topological space is a $Q$-set if every subset is a $G_{\delta}$-set. His reason for asking this question was because in a certain argument involving a topological space $\Psi(\mathcal{A})$ built from a MAD family it would have been helpful to assume that a MAD family cannot be a Q-set. Szeptycki [11] contains some results on van Douwen's $\Psi$ and also on Q-sets.

Our construction is similar to that in Fleissner and Miller [3] where a Q-set is obtained which is concentrated on the rationals. In Judah and Shelah [7] it is shown consistent to have a Q-set while at the same time $\mathfrak{b}=\mathfrak{d}=\omega_{1}$. Their Q-set forcing has the Sacks property. Their forcing is also used in Nowik and Weiss [10] to construct a Q-set with certain properties and also in Gruenhage and Koszmider [4] to construct a topological space with certain properties. In our model as in [3] we have $\mathfrak{d}=\mathfrak{c}=\omega_{2}$ and $\mathfrak{b}=\omega_{1}$.

In Dow [2] and Brendle [1] a type of Q-set forcing is used which preserves towers $\left(\right.$ so $\left.\mathfrak{p}=\omega_{1}\right)$ and which generalizes Hechler dominating real forcing, and $\mathfrak{b}=\mathfrak{d}=\mathfrak{c}$.

THEOREM 1. It is relatively consistent with ZFC that there exists a MAD family $\mathcal{A} \subseteq[\omega]^{\omega}$ which is also a $Q$-set.

2000 Mathematics Subject Classification: Primary 03E35.

Thanks to the Fields Institute for Research in Mathematical Sciences at the University of Toronto for their support during the time this paper was written and to Juris Steprāns who directed the special program in set theory and analysis. 
Proof. We begin by forcing a generic MAD family and then we iterate our Q-set forcing to make the generic MAD into a Q-set. The difficulty is to ensure the family stays maximal.

Let $\mathbb{P}$ be the usual poset for forcing a MAD family: $(p, q) \in \mathbb{P}$ iff

(1) $p: F \rightarrow 2^{N}$ for some finite $F \subseteq \omega_{1}$ and $N<\omega$ (write $F=\operatorname{dom}(p)$ and $N=N_{p}$ ),

(2) $q$ is a partial function from a subset of $[F]^{2}$ into $N$,

(3) if $q(\alpha, \beta)=n$, then for every $i$ with $n \leq i<N$ either $p(\alpha)(i)=0$ or $p(\beta)(i)=0$.

The uniformity $N$ of lengths in condition (2) is not strictly necessary but it will be convenient and would occur on a dense set anyway.

Define $\left(p_{1}, q_{1}\right) \leq\left(p_{2}, q_{2}\right)$ iff

(1) $\operatorname{dom}\left(p_{1}\right) \supseteq \operatorname{dom}\left(p_{2}\right)$,

(2) $p_{1}(\alpha) \supseteq p_{2}(\alpha)$ for all $\alpha \in \operatorname{dom}\left(p_{2}\right)$,

(3) $q_{1} \supseteq q_{2}$.

Intuitively, we are describing a family $\left\{a_{\alpha} \subseteq \omega: \alpha<\omega_{1}\right\}$ as follows:

(1) $p(\alpha)=s$ means $\left(i \in a_{\alpha}\right.$ iff $\left.s(i)=1\right)$ for $i<|s|$,

(2) $q(\alpha, \beta)=n$ promises that $a_{\alpha} \cap a_{\beta} \subseteq n$.

Note that $\left(p_{1}, q_{1}\right)$ and $\left(p_{2}, q_{2}\right)$ are compatible iff there exists $p_{3} \leq p_{1}, p_{2}$ such that $\left(p_{3}, q_{1} \cup q_{2}\right)$ is in $\mathbb{P}$.

This forcing is due to Hechler [5]. For $G \mathbb{P}$-generic over $M$ define

$$
x_{\alpha}^{G}=\bigcup\{p(\alpha): \exists q(p, q) \in G\} .
$$

Let $X=\left\{x_{\alpha}^{G}: \alpha<\omega_{1}\right\}$ and let $\mathcal{A}=\left\{a_{\alpha} \subseteq \omega: \alpha<\omega_{1}\right\}$ where each $x_{\alpha}$ is the characteristic function of $a_{\alpha}$, i.e.

$$
a_{\alpha}=\left\{n: x_{\alpha}(n)=1\right\} \text {. }
$$

The following lemma is due to Hechler.

Lemma 2. $\mathbb{P}$ is ccc. If $G$ is $\mathbb{P}$-generic over $M$, then in $M[G]$ the set $\mathcal{A}$ is a maximal almost disjoint family of infinite subsets of $\omega$.

We will in a sense need to reprove this lemma since we will show that after our new version of Q-set forcing our generic family still remains a maximal almost disjoint family. The idea of the argument is that given a name $\tau$ for some infinite subset of $\omega$, we find an $\alpha$ which is not involved in deciding $n \in \tau$ for any $n$. Then we get a contradiction by swapping the value of $x_{\alpha}(n)=0$ to $x_{\alpha}^{\prime}(n)=1$ while still forcing $n \in \tau$. In the usual Q-set forcing, while the condition forcing $n \in \tau$ does not directly talk about $x_{\alpha}$, it may decide that $[s] \subseteq U_{n}$ where the other condition says $x_{\alpha} \notin U_{n}$. These conditions may become inconsistent when we change to $x_{\alpha}^{\prime}$ because it might be that $s \subseteq x_{\alpha}^{\prime}$ even though $s$ is not a subset of $x_{\alpha}$. 
A new Q-set forcing. The following is to motivate our definition of $\mathbb{P} * \widetilde{\mathbb{Q}}$. It would be the definition of the new Q-set forcing in the model $M[G]$ where $G$ is $\mathbb{P}$-generic.

For $x \in 2^{\omega}, s \in 2^{<\omega}, k<\omega$ define

$$
\operatorname{swap}(x, s, k)=\left\{y \in 2^{\omega}: s \subseteq y,|\{i \geq|s|: y(i) \neq x(i)\}| \leq k\right\} .
$$

Note that $\operatorname{swap}(x, s, k)$ is a countable closed subset of $[s]$. It contains $x$ if $s \subseteq x$. Also $\operatorname{swap}(x,\langle\rangle, 0)=\{x\}$.

Suppose we are given $X \subseteq 2^{\omega}$ such that for all $x \neq y \in X$ there are infinitely many $n$ with $x(n) \neq y(n)$. For $Y \subseteq X$ define $\mathbb{Q}(X, Y)$ as follows: $r \in \mathbb{Q}(X, Y)$ iff $r$ is a finite subset of

$$
\left\{(n, s): n<\omega, s \in 2^{<\omega}\right\} \cup\left\{(n,(x, t, k)): x \in Y, t \in 2^{<\omega}, n, k<\omega\right\}
$$

subject to the condition:

$$
\text { if }(n, s) \in r \text { and }(n,(x, t, k)) \in r \text {, then }[s] \cap \operatorname{swap}(x, t, k)=\emptyset .
$$

The ordering is by inclusion: $r_{1} \leq r_{2}$ iff $r_{1} \supseteq r_{2}$. The meaning of these conditions is:

(1) $(n, s)$ means " $[s] \subseteq U_{n}$ ",

(2) $(n,(x, t, k))$ means "swap $(x, t, k) \cap U_{n}=\emptyset "$.

Now suppose $G$ is $\mathbb{Q}(X, Y)$-generic over a model $N$. Define

$$
U_{n}^{G}=\bigcup\{[s]: \exists r \in G(n, s) \in r\} .
$$

An easy genericity argument shows that

$$
X \cap \bigcap_{n<\omega} U_{n}^{G}=X \backslash Y .
$$

To see this suppose $y \in Y$ and $r$ is any condition. Let $n$ be sufficiently large so as to not appear in $r$ at all. Then let $r^{\prime}=r \cup\{(n,(y,\langle\rangle, 0))\}$ and note that

$$
r^{\prime} \Vdash y \notin U_{n} .
$$

On the other hand let $y \in X \backslash Y, r$ be any condition, and $n<\omega$ be arbitrary. Since $y$ is infinitely often different from any element of $X$ mentioned in $r$ (they must come from $Y$ ), we can find $l<\omega$ so that

$$
[y\lceil l] \cap \operatorname{swap}(x, s, k)=\emptyset
$$

for any $(n,(x, s, k)) \in r$. Now we let $r^{\prime}=r \cup\{(n, y\lceil l)\}$. Then

$$
r^{\prime} \Vdash y \in U_{n} \text {. }
$$

Next we describe the ordering $\mathbb{P} * \widetilde{\mathbb{Q}}$ which is a basic building block of our iteration. If $G$ is $\mathbb{P}$-generic over $M$ then $\widetilde{\mathbb{Q}}^{G}$ is essentially the same as $\mathbb{Q}(X, X)$.

Define $((p, q), r) \in \mathbb{P} * \widetilde{\mathbb{Q}}$ iff 
(1) $(p, q) \in \mathbb{P}$,

(2) $r$ is a finite subset of the union of

$$
\left\{(n, t): n<N_{p}, t \in 2^{<N_{p}}\right\}
$$

and

$$
\left\{(n,(\alpha, s, k)): \alpha \in \operatorname{dom}(p), s \in 2^{<N_{p}}, n, k<N_{p}\right\},
$$

(3) if $(n,(\alpha, s, k)) \in r$ and $(n, t) \in r$, then either $s$ and $t$ are incomparable or $s \subseteq t$ and

$$
|\{i:|s| \leq i<|t|, t(i) \neq p(\alpha)(i)\}|>k .
$$

Condition (3) guarantees that for any $x \in 2^{\omega}$ such that $x \supseteq p(\alpha)$ we have

$$
\operatorname{swap}(x, s, k) \cap[t]=\emptyset .
$$

The ordering is given by

$$
\left(\left(p_{1}, q_{1}\right), r_{1}\right) \leq\left(\left(p_{2}, q_{2}\right), r_{2}\right) \quad \text { iff } \quad\left(p_{1}, q_{1}\right) \leq\left(p_{2}, q_{2}\right) \text { and } r_{1} \supseteq r_{2} .
$$

Note that $\left(\left(p_{1}, q_{1}\right), r_{1}\right)$ and $\left(\left(p_{2}, q_{2}\right), r_{2}\right)$ are compatible iff there exists $p_{3} \leq$ $p_{1}, p_{2}$ such that $\left(\left(p_{3}, q_{1} \cup q_{2}\right), r_{1} \cup r_{2}\right)$ is a condition.

The $\omega_{2}$ iteration. Our iteration can be described as a suborder of the product

$$
\mathbb{P} \times \sum_{\alpha<\omega_{2}} \mathbb{E}
$$

where $\mathbb{E}$ is the set of all finite subsets of

$$
\left\{(n, t): n<\omega, t \in 2^{<\omega}\right\} \cup\left\{(n,(\alpha, s, k)): \alpha \in \omega_{1}, s \in 2^{<\omega}, n, k<\omega\right\}
$$

and $\sum_{\alpha<\omega_{2}} \mathbb{E}$ is the set of all $r: \omega_{2} \rightarrow \mathbb{E}$ such that $r(\delta)$ is trivial (i.e., the empty set) for all but finitely many $\delta$.

By induction on $\beta \leq \omega_{2}$ define

$$
\mathbb{P}_{\beta} \subseteq \mathbb{P} \times \sum_{\alpha<\beta} \mathbb{E}
$$

as follows. Set $\mathbb{P}_{0}=\mathbb{P}$, and suppose that we have defined $\mathbb{P}_{\beta}$ and we are also given a $\mathbb{P}_{\beta}$ name $Y_{\beta}$ for a subset of $\omega_{1}$, i.e.,

$$
\Vdash_{\beta} Y_{\beta} \subseteq \omega_{1} .
$$

Define $((p, q), r) \in \mathbb{P}_{\beta+1}$ iff

(1) $\left((p, q), r\lceil\beta) \in \mathbb{P}_{\beta}\right.$,

(2) $((p, q), r(\beta)) \in \mathbb{P} * \widetilde{\mathbb{Q}}$,

(3) $\left((p, q), r\lceil\beta) \Vdash_{\beta} \alpha \in Y_{\beta}\right.$ whenever $(n,(\alpha, s, k)) \in r(\beta)$ for some $n, s, k, \alpha$.

For limit ordinals $\lambda \leq \omega_{2}$ we define $((p, q), r) \in \mathbb{P}_{\lambda}$ iff for all $\beta<\lambda$ we have $\left((p, q), r\lceil\beta) \in \mathbb{P}_{\beta}\right.$, and $r(\beta)$ is the trivial condition (i.e. empty set) for all but finitely many $\beta<\lambda$. 
Since the iteration of a ccc forcing is ccc, all of these forcings are ccc. To see this directly we can argue as follows: Standard arguments using $\Delta$ systems show that $\mathbb{P}_{\beta}$ has precaliber $\omega_{1}$, i.e., any $\omega_{1}$ sequence of conditions contains an $\omega_{1}$ subsequence which is centered. Start with $\left(\left(p_{\alpha}, q_{\alpha}\right), r_{\alpha}\right) \in \mathbb{P}_{\beta}$ for $\alpha<\omega_{1}$. We can find an uncountable $\Sigma \subseteq \omega_{1}$ and finite sets $F$ and $H$ and $N<\omega$ so that:

(1) $N_{\alpha}=N$ for all $\alpha \in \Sigma$,

(2) $\operatorname{dom}\left(p_{\alpha}\right) \cap \operatorname{dom}\left(p_{\beta}\right)=F$ for $\alpha \neq \beta \in \Sigma$,

(3) $\operatorname{dom}\left(r_{\alpha}\right) \cap \operatorname{dom}\left(r_{\beta}\right)=H$ for $\alpha \neq \beta \in \Sigma$,

(4) $p_{\alpha}\lceil F$ are all the same for $\alpha \in \Sigma$,

(5) $q_{\alpha}\left\lceil[F]^{2}\right.$ are all the same for $\alpha \in \Sigma$,

(6) $r_{\alpha}\left\lceil H\right.$ are all the same with respect to $\left\{(n, s): n<\omega, s \in 2^{<\omega}\right\}$ for $\alpha \in \Sigma$.

Then any two (or even finite subset) of them are compatible.

Assuming that the ground model satisfies the $\mathrm{GCH}$, by the usual book keeping argument we can arrange things so that for any $Y \subseteq \omega_{1}$ which appears in $M\left[G_{\omega_{2}}\right]$ there will be a name for it in the list $Y_{\alpha}$ for some $\alpha<\omega_{2}$. The simplest way to do this is to take

$$
\left\{\left\langle Z_{\beta}^{\alpha}: \beta<\omega_{1}\right\rangle: \alpha<\omega_{2}\right\}
$$

which lists all $\omega_{1}$ sequences of countable subsets of $\mathbb{P} \times \sum_{\alpha<\omega_{2}} \mathbb{E}$ with $\omega_{2}$ repetitions and then define

$$
Y_{\alpha}=\left\{\langle p, \check{\beta}\rangle: \beta<\omega_{1}, p \in Z_{\beta}^{\alpha} \cap \mathbb{P}_{\alpha}\right\}
$$

If we define

$$
x_{\alpha}=\bigcup\left\{s \in 2^{<\omega}: \exists((p, q), r) \in G s=p(\alpha)\right\}, \quad X=\left\{x_{\alpha}: \alpha<\omega_{1}\right\},
$$

then $X$ will be the characteristic functions of an almost disjoint family $\mathcal{A}=$ $\left\{a_{\alpha}: \alpha<\omega_{1}\right\}$. Furthermore if we define the open sets

$$
U_{n}^{\beta}=\bigcup\{[s]: \exists((p, q), r) \in G(n, s) \in r(\beta)\}
$$

then by the usual genericity argument

$$
\bigcap_{n<\omega} U_{n}^{\beta} \cap X=\left\{x_{\alpha}: \alpha \notin Y_{\beta}^{G}\right\}
$$

and so $X$ will be a $Q$-set.

The nontrivial part of our argument is to prove that $\mathcal{A}$ remains a maximal almost disjoint family. So let $\tau$ be a name for a counterexample, i.e., suppose

$$
\left(\left(p_{0}, q_{0}\right), r_{0}\right) \Vdash \tau \in[\omega]^{\omega} \text { and } \forall \alpha<\omega_{1} \tau \cap a_{\alpha} \text { is finite. }
$$

Let $\Sigma \subseteq \mathbb{P}_{\omega_{2}}$ be a countable set of conditions extending $\left(\left(p_{0}, q_{0}\right), r_{0}\right)$ such that for any $n \in \omega, \Sigma$ contains a maximal antichain beneath $\left(\left(p_{0}, q_{0}\right), r_{0}\right)$ 
which decides $n \in \tau$. Let $\alpha_{0}<\omega_{1}$ be any ordinal not mentioned in any condition from $\Sigma$. We show $a_{\alpha_{0}} \cap \tau$ is infinite.

Suppose for contradiction that we have $\left(\left(p_{1}, q_{1}\right), r_{1}\right) \leq\left(\left(p_{0}, q_{0}\right), r_{0}\right)$ and $N_{1}<\omega$ such that

$$
\left(\left(p_{1}, q_{1}\right), r_{1}\right) \Vdash \tau \cap a_{\alpha_{0}} \subseteq N_{1} .
$$

Without loss of generality we may assume that $N_{1}=N_{p_{1}}$. By tacking on strings of zeros to the conditions in $p_{1}$ we may assume that every integer occurring in $r$ is bounded by $N_{1}-2$ (and not just as required by $N_{1}$ ). Let

$$
F=\left\{\beta:\left\{\alpha_{0}, \beta\right\} \in \operatorname{dom}\left(q_{1}\right)\right\} .
$$

Define $r^{\prime} \supseteq r_{1}$ as follows:

$$
r^{\prime}(\delta)=r_{1}(\delta) \cup\left\{\left(n,\left(\alpha_{0}, t^{\prime}, k+1\right)\right):\left(n,\left(\alpha_{0}, t, k\right)\right) \in r_{1}(\delta), t^{\prime} \in A_{\delta, n}, t^{\prime} \supseteq t\right\}
$$

for each $\delta$ where

$$
A_{\delta, n}=\left\{t^{\prime} \in 2^{N_{1}-1}: t^{\prime} \text { is incomparable with all } s \text { such that }(n, s) \in r_{1}(\delta)\right\} .
$$

Note that $\left(\left(p_{1}, q_{1}\right), r^{\prime}\right)$ is a valid condition because $\alpha_{0}$ is forced into $Y_{\delta}$ and $t^{\prime}$ incomparable with all $s$ which might be a problem. Let $G$ be a generic filter containing $\left(\left(p_{1}, q_{1}\right), r^{\prime}\right)$. Since $\tau^{G}$ is almost disjoint from each $a_{\beta}^{G}$ and infinite, there exists some $n_{0} \in \tau^{G}$ with $n_{0}>N_{1}$ and $n_{0} \notin a_{\beta}^{G}$ for all $\beta \in F$. Let $\left(\left(p_{2}, q_{2}\right), r_{2}\right) \in \Sigma \cap G$ be so that

$$
\left(\left(p_{2}, q_{2}\right), r_{2}\right) \Vdash n_{0} \in \tau .
$$

Since it is from $\Sigma$ it does not mention $\alpha_{0}$.

Let $\left(\left(p^{*}, q^{*}\right), r^{*}\right) \in G$ be stronger than both $\left(\left(p_{1}, q_{1}\right), r^{\prime}\right)$ and $\left.\left(p_{2}, q_{2}\right), r_{2}\right)$ and such that $N^{*}>n_{0}$. Note that $\left(\left(p^{*}, q_{1} \cup q_{2}\right), r^{\prime} \cup r_{2}\right)$ is a valid condition. Any $\gamma$ that needs to be forced into some $Y_{\beta}$ is already forced in by either $\left(\left(p_{1}, q_{1}\right), r^{\prime} \uparrow \beta\right)$ or $\left(\left(p_{2}, q_{2}\right), r_{2} \uparrow \beta\right)$.

If $p^{*}\left(\alpha_{0}\right)\left(n_{0}\right)=1$ then we already have a contradiction and there is nothing to prove. So assume not, and define $p^{\prime}$ to be exactly the same as $p^{*}$ except $p^{\prime}\left(\alpha_{0}\right)\left(n_{0}\right)=1$.

Claim. $\left(\left(p^{\prime}, q_{1} \cup q_{2}\right), r \cup r_{2}\right)$ is a valid condition, extending both $\left(\left(p_{1}, q_{1}\right), r_{1}\right)$ and $\left(\left(p_{2}, q_{2}\right), r_{2}\right)$.

Proof. Note that we have dropped the extra conditions from $r^{\prime}$, these were put there just to prove this Claim. The fact that $p^{\prime}$ extends both $p_{1}$ and $p_{2}$ uses the fact that $n_{0}>N_{p_{1}}=N_{1}$ and $\alpha_{0}$ is not in the domain of $p_{2}$. Similarly since $q_{2}$ does not mention $\alpha_{0}$, it follows that if $\left\{\alpha_{0}, \beta\right\} \in$ $\operatorname{dom}\left(q_{1} \cup q_{2}\right)$, then $\beta \in F$ and we know that $p^{*}(\beta)(n)=0$ for each $\beta \in F$. So making $p^{\prime}\left(\alpha_{0}\right)(n)=1$ does not violate any promises of disjointness made in $q_{1} \cup q_{2}$. So we have $\left(p^{\prime}, q_{1} \cup q_{2}\right) \in \mathbb{P}$.

Now fix $\delta$; we must check that

$$
\left(\left(p^{\prime}, q_{1} \cup q_{2}\right), r_{1}(\delta) \cup r_{2}(\delta)\right) \in \mathbb{P} * \widetilde{\mathbb{Q}} .
$$


We need to check condition (3) in the definition of $\mathbb{P} * \widetilde{\mathbb{Q}}:$ if $(n,(\alpha, s, k)),(n, t)$ $\in r_{1}(\delta) \cup r_{2}(\delta)$ then either $s$ and $t$ are incomparable or $s \subseteq t$ and

$$
\left|\left\{i:|s| \leq i<|t|, t(i) \neq p^{\prime}(\alpha)(i)\right\}\right|>k .
$$

Suppose it fails. It can only fail if $\alpha=\alpha_{0}$ and since $r_{2}$ does not mention $\alpha_{0}$ it must be that $\left(n,\left(\alpha_{0}, s, k\right)\right) \in r_{1}(\delta)$ and $(n, t) \in r_{2}(\delta)$. Also it must be that $s$ and $t$ are comparable with $s \subseteq t$ but

$$
\left|\left\{i:|s| \leq i<|t|, t(i) \neq p^{\prime}\left(\alpha_{0}\right)(i)\right\}\right| \leq k
$$

Note also that $|t|>n_{0}>N_{1}$ because otherwise

$$
\left\{i:|s| \leq i<|t|, t(i) \neq p^{\prime}\left(\alpha_{0}\right)(i)\right\}=\left\{i:|s| \leq i<|t|, t(i) \neq p^{*}\left(\alpha_{0}\right)(i)\right\} .
$$

But then

$$
\left|\left\{i:|s| \leq i<|t|, t(i) \neq p^{*}\left(\alpha_{0}\right)(i)\right\}\right|>k
$$

because $\left(\left(p^{*}, q_{1} \cup q_{2}\right), r_{1}(\delta) \cup r_{2}(\delta)\right) \in \mathbb{P} * \widetilde{\mathbb{Q}}$.

Now let $t^{\prime}=t \uparrow\left(N_{1}-1\right)$.

CASE 1: $t^{\prime}$ is comparable with some $s^{\prime}$ such that $\left(n, s^{\prime}\right) \in r_{1}(\delta)$. Recall that every integer occurring in $r_{1}(\delta)$ is bounded by $N_{1}-1$. So it must be that $s^{\prime} \subseteq t^{\prime}$ but intuitively this is easy because $r_{1}(\delta)$ is already asserting $\left[s^{\prime}\right] \subseteq U_{n}^{\bar{\delta}}$ and this implies $[t] \subseteq U_{n}^{\delta}$. More formally, $s^{\prime} \subseteq t^{\prime}$ and therefore $s^{\prime}$ and $s$ are both initial strings of $t^{\prime}$ and so comparable; but then we know that

$$
\left|\left\{i:|s| \leq i<\left|s^{\prime}\right|<N_{1}, s^{\prime}(i) \neq p_{1}\left(\alpha_{0}\right)(i)\right\}\right|>k .
$$

But this is still true for $p^{\prime}$ since we have not changed it below $N_{1}$.

CASE 2: $t^{\prime} \in A_{\delta, n}$ and so we added $\left(\alpha_{0}, t^{\prime}, k+1\right)$ to $r^{\prime}(\delta)$. But remember $\left(\left(p^{*}, q_{1} \cup q_{2}\right), r^{\prime} \cup r_{2}\right)$ is a valid condition, which means that

$$
\left|\left\{i: N_{1} \leq i<|t|, t(i) \neq p^{*}\left(\alpha_{0}\right)(i)\right\}\right|>k+1
$$

but $k<N_{1}-2$ and $p^{*}\left(\alpha_{0}\right)$ agrees with $p^{\prime}\left(\alpha_{0}\right)$ except at exactly one coordinate so

$$
\left|\left\{i:|s|<N \leq i<|t|, t(i) \neq p^{\prime}\left(\alpha_{0}\right)(i)\right\}\right|>k .
$$

This proves that $\left(\left(p^{\prime}, q_{1} \cup q_{2}\right), r_{1}(\delta) \cup r_{2}(\delta)\right) \in \mathbb{P} * \widetilde{\mathbb{Q}}$ for every $\delta$.

Finally we must show that

$$
\left(\left(p^{\prime}, q_{1} \cup q_{2}\right),\left(r_{1} \cup r_{2}\right)\lceil\beta) \Vdash_{\beta} \gamma \in Y_{\beta}\right.
$$

whenever $(n,(\gamma, s, k)) \in\left(r_{1} \cup r_{2}\right)(\beta)$ for some $n, s, k$. But by induction

$$
\left(\left(p^{\prime}, q_{1} \cup q_{2}\right),\left(r_{1} \cup r_{2}\right)\lceil\beta)\right.
$$

extends both $\left(\left(p_{1}, q_{1}\right), r_{1} \uparrow \beta\right)$ and $\left(\left(p_{2}, q_{2}\right), r_{2} \uparrow \beta\right)$, one of which does the required forcing. 


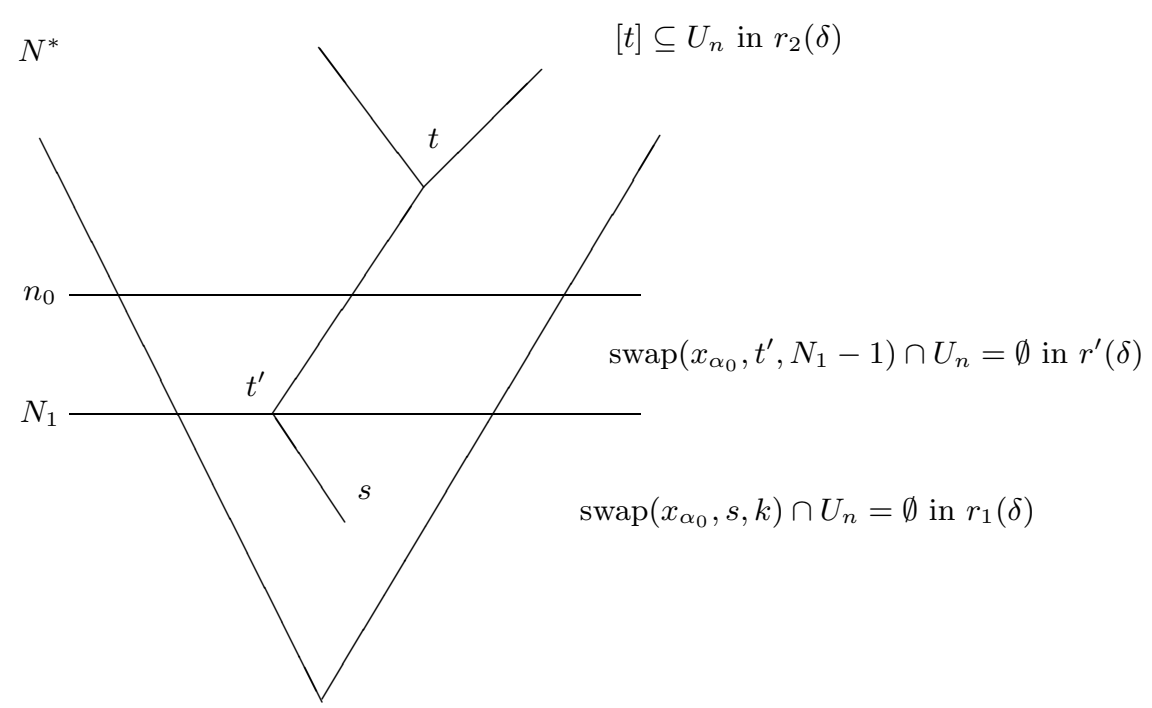

Fig. 1. The swap

This proves the Claim. The theorem now follows from the contradiction that

$$
\begin{aligned}
& \left(\left(p_{1}, q_{1}\right), r_{1}\right) \Vdash \tau \cap a_{\alpha_{0}} \subseteq N_{1}, \\
& \left(\left(p_{2}, q_{2}\right), r_{2}\right) \Vdash n_{0} \in \tau,
\end{aligned}
$$

where $n_{0}>N_{1}$ and

$$
\left(\left(p^{\prime}, q_{1} \cup q_{2}\right), r_{1} \cup r_{2}\right) \Vdash n_{0} \in a_{\alpha_{0}} .
$$

REMARK. The usual Q-set forcing kills the maximality of an almost disjoint family $X$. To see this suppose $\left\{x_{n}: n<\omega\right\} \subseteq X$ and conditions are finite consistent sets of sentences of the form " $[s] \subseteq U_{n}$ " or " $x \notin U_{n}$ " where $x \in X \backslash\left\{x_{n}: n<\omega\right\}$. So when we force we get a $G_{\delta}$-set so that

$$
\bigcap_{n<\omega} U_{n} \cap X=\left\{x_{n}: n<\omega\right\}
$$

In the generic extension we can find $\left\{k_{n}: n<\omega\right\}$ increasing so that

$$
k_{n+1} \notin \bigcup_{i<n} x_{i}, \quad\left\{y \subseteq \omega: k_{n+1} \in y\right\} \subseteq \bigcap_{i<n} U_{i} .
$$

Why? Given $p$ find $k_{n+1}>k_{n}$ not in any $x_{i}$ for $i<n$ or in any $x$ mentioned in $p$ and put

$$
p^{\prime}=p \cup\left\{\left[s_{1}\right] \subseteq U_{i}: i<n, s \in 2^{k_{n+1}-1}\right\} .
$$

But then $\left\{k_{n}: n<\omega\right\}$ is almost disjoint from all elements of $X$.

REMARK. Since there are perfect almost disjoint families, e.g.,

$$
\mathcal{A}=\left\{\{x \mid n: n<\omega\}: x \in 2^{\omega}\right\} \subset P\left(2^{<\omega}\right),
$$


there are always MAD families of arbitrarily large Borel order. Obviously a Q-set cannot have cardinality continuum, but a $\sigma$-set can.

Define $X \subseteq 2^{\omega}$ to be a $\sigma$-set iff for every Borel set $B \subseteq 2^{\omega}$ there exists a $G_{\delta}$-set $G$ such that $B \cap X=G \cap X$.

A Sierpiński set is an example of $\sigma$-set (Poprougenko, see Miller [9]).

THEOREM 3. It is consistent with any cardinal arithmetic that there exists a MAD $\sigma$-set of size continuum.

Proof. This is an easy modification of the argument of the main theorem. Taking any countable transitive model $M$ first force a generic MAD of size continuum, then do a finite support iteration of length continuum to make it into a $\sigma$-set.

REmark. H. Woodin (see Larson [8]) has shown that if there exists a measurable Woodin cardinal $\kappa$, and $V$ and $V[G]$ are both models of $\mathrm{CH}$ where $V[G]$ is a generic extension using a partial order of size less than $\kappa$, then $V$ and $V[G]$ model exactly the same $\Sigma_{1}^{2}$ sentences. The existence of a MAD $\sigma$-set is a $\Sigma_{1}^{2}$ sentence. It follows that $\mathrm{CH}+$ there exists a measurable Woodin cardinal implies there is a MAD $\sigma$-set.

It is virtually certain that MAD $\sigma$-sets have nothing to do with large cardinals, so we have the conjecture:

ConjeCture 4. CH implies there exists a MAD $\sigma$-set.

ThEOREM 5. The generic $M A D$ set $\mathcal{A}=\left\{a_{\alpha}: \alpha<\omega\right\}$ is concentrated on $\left\{a_{n}: n<\omega\right\}$, i.e., every open set containing $\left\{a_{n}: n<\omega\right\}$ contains all but countably many elements of $\mathcal{A}$.

Proof. Let $M$ be a countable standard model of ZFC and $G$ be $\mathbb{P}$-generic over $M$. Working in $M$ suppose

$$
\Vdash\left\{a_{n}: n<\omega\right\} \subseteq U \text {, an open set. }
$$

Let $\Sigma \subseteq \mathbb{P}$ be countable so that for every $s \in 2^{<\omega}$ there exists a maximal antichain in $\Sigma$ which decides " $[s] \subseteq U$ ".

ClaIM. $\Vdash a_{\alpha} \in U$ for any $\alpha$ larger than any mentioned in $\Sigma$.

Proof. Suppose not and let $(p, q) \Vdash a_{\alpha} \notin U$. Choose some $n$ so that $n$ is not in the domain of $p$. Let $p^{\prime}=p \cup\{(n, s)\}$ where $(\alpha, s) \in p$ and let

$$
q^{\prime}=q \cup \bigcup\{(\{n, \beta\}, k):(\{\alpha, \beta\}, k) \in q\}
$$

so $\left(p^{\prime}, q^{\prime}\right) \leq(p, q)$ and it says the same things about $a_{n}$ and $a_{\alpha}$. There exists $(\widehat{p}, \widehat{q}) \in \Sigma$ compatible with $\left(p^{\prime}, q^{\prime}\right)$ such that $N_{\widehat{p}}>N_{p}$ and

$$
(\widehat{p}, \widehat{q}) \Vdash\left[x_{n}\left\lceil N_{\widehat{p}}\right] \subseteq U .\right.
$$

Let $\left(p^{*}, q^{*}\right)$ extend both $\left(p^{\prime}, q^{\prime}\right)$ and $(\widehat{p}, \widehat{q})$. Change $p^{*}$ to $r$ with the same domain but $r(\alpha)=p^{*}(n)$ and other coordinates all the same. But then 
$\left(r, q^{\prime} \cup \widehat{q}\right)$ is a common extension of both $\left(p^{\prime}, q^{\prime}\right)$ and $(\widehat{p}, \widehat{q})$. And this is a contradiction.

This proves the Claim and Theorem.

THEOREM 6. CH implies there exists a MAD family which is concentrated on the finite subsets of $\omega$ and is a $\lambda$-set (i.e., every countable subset is a relative $G_{\delta}$ ).

Proof. It is easy to construct a MAD family $\left\{a_{\alpha}: \alpha<\omega_{1}\right\}$ so that if $f_{\alpha}: \omega \rightarrow a_{\alpha}$ is the strictly increasing enumeration of $\alpha$, then for every $\alpha<\beta$ we have $f_{\alpha}<^{*} f_{\beta}$ and for every $g \in \omega^{\omega}$ there exists $\alpha<\omega_{1}$ such that $g \leq^{*} f_{\alpha}$, i.e., they form a scale. Rothberger (see Miller [9]) showed that any well-ordered subset of $\left(\omega^{\omega}, \leq^{*}\right)$ is a $\lambda$-set and that any $\omega_{1}$-ordered unbounded set is concentrated on the rationals.

The same large cardinal results lead to the following conjecture:

Conjecture 7. CH implies there exists a MAD family which is concentrated on a countable subset of itself.

Paul Szeptycki pointed out that the Q-set forcing used in Theorem 1 can be used to prove the following:

THEOREM 8. It is relatively consistent that there exists a $Q$-set $X \subseteq[\omega]^{\omega}$ with the property that for every $a \in[\omega]^{\omega}$ for all but countably many $x \in X$ we have $|x \cap a|=|x \backslash a|=\omega$, i.e., $X$ is a strong splitting family.

Proof. We replace $\mathbb{P}$ by the Cohen real partial order, i.e., just drop the $q$ 's from the $(p, q)$. We use the same $\mathbb{P} * \widetilde{\mathbb{Q}}$. Note that in the basic argument for $p^{\prime}$ we could have flipped $p^{\prime}\left(\alpha_{0}\right)\left(n_{0}\right)=1-p^{*}\left(\alpha_{0}\right)\left(n_{0}\right)$ and $\alpha_{0}$ could be any $\alpha<\omega_{1}$ not mentioned in $\Sigma$.

Alan Dow asked if it is possible to have $X=\left\{x_{\alpha} \in 2^{\omega}: \alpha<\omega_{1}\right\}$ and $Y=\left\{y_{\alpha} \in 2^{\omega}: \alpha<\omega_{1}\right\}$ such that $x_{\alpha}={ }^{*} y_{\alpha}$ for every $\alpha<\omega_{1}$, and $X$ a Q-set and $Y$ not a $\mathrm{Q}$-set. The answer is yes, in fact, we force something stronger:

TheOREM 9. It is relatively consistent to have $X=\left\{x_{\alpha} \in 2^{\omega}: \alpha<\omega_{1}\right\}$ and $\left\{y_{n} \in 2^{\omega}: n<\omega\right\}$ such that $x_{n}=^{*} y_{n}$ for every $n<\omega$, and $X$ a $Q$-set concentrated on $\left\{y_{n}: n<\omega\right\}$ (hence $Y=\left\{y_{n}: n<\omega\right\} \cup\left\{x_{\alpha}: \omega \leq \alpha<\omega_{1}\right\}$ is not a $Q$-set).

Proof. This is a variant of the forcing used in Fleissner-Miller [3]. In that forcing we start by adding an $\omega_{1}$ batch of Cohen reals $X=\left\{x_{\alpha} \in 2^{\omega}\right.$ : $\left.\alpha<\omega_{1}\right\}$. The Q-set forcing is modified to always allow statements of the form $e \notin U_{n}^{\alpha}$ where $e$ is any eventually zero element of $2^{\omega}$. With this modification the $\mathrm{Q}$-set $X$ is shown to be concentrated on the eventually zero elements of $2^{\omega}$.

We modify this slightly as follows. Let $y_{n}$ be defined by $y_{n}(m)=x_{n}(m)$ except $y_{n}(n)=1-x_{n}(n)$, i.e., we change only one coordinate. Now in the 
inductive construction of $\mathbb{P}_{\alpha}$ we use the $y_{n}$ 's instead of the eventually zero reals, i.e., if $x \notin U_{m}^{\alpha} \in p(\alpha)$, then either $x=y_{n}$ for some $n$ or $p\left\lceil\alpha \Vdash x \in Y_{\alpha}\right.$. The rest of the argument is the same as in [3].

I am not sure how to do Theorem 9 with a MAD family. This would be interesting because it would show that the $\Psi$ space does not determine whether or not you have a Q-set.

\section{References}

[1] J. Brendle, Dow's principle and Q-sets, Canad. Math. Bull. 42 (1999), 13-24.

[2] A. Dow, On compact separable radial spaces, ibid. 40 (1997), 422-432.

[3] W. G. Fleissner and A. W. Miller, On Q-sets, Proc. Amer. Math. Soc. 78 (1980), 280-284.

[4] G. Gruenhage and P. Koszmider, The Arkhangel'skiu-Tall problem: a consistent counterexample, Fund. Math. 149 (1996), 143-166.

[5] S. H. Hechler, Short complete nested sequences in $\beta N \backslash N$ and small maximal almostdisjoint families, General Topology Appl. 2 (1972), 139-149.

[6] M. Hrušák, MAD families and the rationals, Comment. Math. Univ. Carolin. 42 (2001), 345-352.

[7] H. Judah and S. Shelah, Q-sets, Sierpiński sets, and rapid filters, Proc. Amer. Math. Soc. 111 (1991), 821-832.

[8] P. Larson, Notes on the stationary tower, to appear.

[9] A. W. Miller, Special subsets of the real line, in: Handbook of Set-Theoretic Topology, North-Holland, Amsterdam, 1984, 201-233.

[10] A. Nowik and T. Weiss, Not every Q-set is perfectly meager in the transitive sense, Proc. Amer. Math. Soc. 128 (2000), 3017-3024.

[11] P. Szeptycki, Countable metacompactness in $\Psi$-spaces, ibid. 120 (1994), 1241-1246.

Department of Mathematics, Van Vleck Hall

University of Wisconsin-Madison

480 Lincoln Drive

Madison, WI 53706-1388, U.S.A.

E-mail: miller@math.wisc.edu

Web: http://www.math.wisc.edu/ ${ }^{\sim}$ miller 